\title{
High Signal Intensity on Diffusion-Weighted Images Reflects Acute Phase of Deep Vein Thrombus
}

\author{
Toshihiro Gi $^{1}$ Yasuyoshi Kuroiwa ${ }^{1,2}$ Atsushi Yamashita ${ }^{1}$ Yuko Mizutani ${ }^{3}$ Taketoshi Asanuma ${ }^{4}$ \\ Tosiaki Miyati $^{5}$ Kazunari Maekawa ${ }^{1}$ Murasaki Aman ${ }^{6}$ Takuroh Imamura ${ }^{7}$ Yujiro Asada ${ }^{1}$
}

\footnotetext{
${ }^{1}$ Department of Pathology, Faculty of Medicine, University of Miyazaki, Miyazaki, Japan

2 Department of Radiological Technology, Koga General Hospital, Miyazaki, Japan

3 Division of Radiology, Department of Veterinary Sciences, Faculty of Agriculture, University of Miyazaki, Miyazaki, Japan

${ }^{4}$ Development of Veterinary Medicine, Faculty of Veterinary Medicine, Imabari Campus, Okayama University of Science, Imabari, Japan

5 Institute of Medical, Pharmaceutical and Health Sciences, Faculty of Health Sciences, Kanazawa University, Ishikawa, Japan

6 Department of Diagnostic Pathology, University of Miyazaki Hospital, Faculty of Medicine, University of Miyazaki, Miyazaki, Japan

7 Department of Internal Medicine, Koga General Hospital, Miyazaki, Japan
}

Thromb Haemost 2020;120:1463-1473.

\author{
Address for correspondence Yujiro Asada, MD, PhD, Department of \\ Pathology, Faculty of Medicine, University of Miyazaki, 5200 Kihara, \\ Kiyotake, Miyazaki 889-1692, Japan \\ (e-mail: yasada@med.miyazaki-u.ac.jp).
}

\begin{abstract}
Keywords

- deep vein thrombosis

- diffusion-weighted imaging

- erythrocyte

- magnetic resonance imaging

- pathology

The effects of antithrombotic therapy on deep vein thrombosis (DVT) can be affected by thrombus age, which cannot be reliably determined by noninvasive imaging modalities. We investigated whether magnetic resonance (MR) diffusion-weighted imaging (DWI) can localize and determine the age of venous thrombus in patients with DVT, animal models, and human blood in vitro. Signal intensity (SI) on DWI and the apparent diffusion coefficient (ADC) of thrombi were assessed in eight patients with DVT using a 1.5-T MR imaging (MRI) system. We assessed the organizing processes as venous thrombus developed in the rabbit jugular vein using a 3.0-T MRI system over time. We also assessed MRI signals of human blood in vitro using the 1.5-T MRI system. Venous thrombi were detected by DWI as areas of high or mixed high and iso SI in all patients. The ADCs were lower in the proximal, than in the distal portion of the thrombi. The thrombi of rabbit jugular veins histologically organized in a timedependent manner, with high SI on DWI at 4 hours, mixed high and iso SI at 1 and 2 weeks, and iso SI at 3 weeks. The ADC correlated negatively with erythrocyte content, and positively with smooth muscle cells, macrophages, hemosiderin, and collagen content. MRI signals of human blood in vitro showed that ADCs were affected by erythrocyte content, but not by blood clotting. MR-DWI can detect venous thrombus, and high SI on DWI accompanied by a low ADC might reflect erythrocyte-rich, acutephase thrombi.
\end{abstract}

received

April 10, 2020

accepted after revision June 10,2020
DOI https://doi.org/ $10.1055 / \mathrm{s}-0040-1714280$ ISSN 0340-6245. (c) 2020 Georg Thieme Verlag KG Stuttgart · New York
License terms

(웅 $\odot \circledast$ 


\section{Introduction}

Venous thrombus consists of blood cells and fibrin that have time-dependent organizing processes, such as endothelialization, macrophage infiltration, myofibroblast migration/ growth, and replacement with fibrous tissue. ${ }^{1}$ Therefore, assessments of venous thrombus age and components are important to provide information that could help to determine the effects of antithrombotic therapy. ${ }^{2-4}$ Ultrasound and contrast-enhanced computerized tomography (CT) are gold standards for a diagnosis of deep vein thrombosis (DVT) in lower extremities. However, ultrasound is operator-dependent and the field of observation is limited. Contrast-enhanced CT has the disadvantages of radiation exposure, allergies to contrast agent, and is contraindicated for patients with chronic renal failure. ${ }^{5}$ A means of determining the age of venous thrombi using CT has not been established.

Magnetic resonance (MR) diffusion-weighted imaging (DWI) is noninvasive, and it relies on detecting the random motion of free water molecules. Although several reports have indicated that MR imaging (MRI) is useful for diagnosing DVT, ${ }^{6-10}$ a method of defining thrombus age has not been established. The present study aimed to determine whether DWI can detect venous thrombi in patients with DVT, and define thrombus age in a rabbit model compared with histological findings.

\section{Methods}

Full description of this section is available in the Supplementary Material (available in the online version).

\section{Clinical Study of Patients with DVT Using Magnetic Resonance Imaging}

The Ethics Committees at the University of Miyazaki and Koga General Hospital approved this retrospective study of eight patients with DVT (University of Miyazaki Study protocol 0-0665, Koga General Hospital Approval no. 16-14). All patients were assessed by MRI, and five of them were treated with a combination of heparin and warfarin for 3 to 28 days before MRI. We diagnosed DVT based on clinical and laboratory findings, and imaging findings of ultrasonography, CT, and/or MRI. Time after onset was estimated as the amount of elapsed time from clinical symptoms to MRI acquisition. D-dimer levels were measured using NANOPIA (Sekisui Medical, Tokyo, Japan), a latex agglutination assay kit. The cut-off value of D-dimer was $1.0 \mu \mathrm{g} / \mathrm{mL} .^{11} \mathrm{D}$-dimer testing and MRI were performed on the same day in three patients, and D-dimer testing was performed at $2(n=1), 4(n=3)$, or 6 days $(n=1)$ before MRI. D-dimer test was performed on two patients under anticoagulant treatment. We acquired MR images using a 1.5-T Signa Excite HDxt superconducting system combined with a 12-channel body-phased array coil (GE Medical Systems, Waukesha, Wisconsin, United States). MRI was performed for all patients in sequences of DWI, T1-weighted images (T1WI), and T2-weighted images (T2WI).

\section{Image and Data Analysis}

Thrombus volumes on T1WI were calculated using OsiriX MD v.10.0.6 32-bit. ${ }^{12}$ We analyzed contrast-to-noise ratios (CNRs) on DWI, T1WI, and T2WI, and apparent diffusion coefficient (ADC) within regions of interest (ROIs) corresponding to thrombi on T1WI axial slices on ADC maps ( $n=5-31$ each) from the patients. Cursors for ROI were placed within thrombus to measure cross-sectional areas and signal intensity (SI) using Image J (U.S. National Institutes of Health, Bethesda, Maryland, United States). We compared these values in slices of thrombi from the inferior vena cava (IVC), pelvic vein ( $n=35$ slices), femoral vein ( $n=36$ slices), and vein in the lower leg ( $n=32$ slices), and in the proximal ( $n=51$ slices) and distal ( $n=52$ slices) areas.

\section{Experimental Animal Study of Venous Thrombus Formation and Magnetic Resonance Imaging in Rabbit} The Animal Care Committees of the participating institutions approved the animal research protocols (Approval No. 2016-509), which conformed to the Guide for the Care and Use of Laboratory Animals published by the U.S. National Institutes of Health. -Supplementary Table S1 and -Supplementary Fig. S1 (available in the online version) show the experimental design, sample number, and the procedure of thrombus formation. Venous thrombi were induced in rabbit right jugular veins by endothelial denudation and blood stasis as described with a minor modification. ${ }^{13}$ The jugular veins were visualized with 3.0-T MRI scanner with a Vantage Titan 3T four-channel flex SPEEDER coil (Toshiba Medical Systems, Tokyo, Japan) at 4 hours and at 1,2 , and 3 weeks after denudation. We localized jugular veins with thrombi on T1WI. We then calculated the ratios of thrombus SI to adjacent muscle tissue SI in DWI, T1WI, and $\mathrm{T} 2 \mathrm{WI}$, and $\mathrm{ADC}$ values in ADC maps within ROI corresponding to the thrombi on T1WI of axial slices ( $n=1-6$ slices each).

\section{Immunohistochemistry of Rabbit Venous Thrombus}

Four percent paraformaldehyde-fixed, paraffin sections of the jugular veins with thrombi were stained with hematoxylin and eosin, Berlin blue (iron deposition), and Sirius red (collagen), and immunostained for platelet glycoprotein IIb/IIIa (Affinity Biologicals Inc., Ancaster, Ontario, Canada), fibrin (Accurate Chemical \& Scientific Corp., Westbury, New York, United States), $\alpha$-smooth muscle actin (mouse monoclonal, clone 1A4; Dako, Glostrup, Denmark), and rabbit macrophages (RAM11, Dako).${ }^{14}$ Positive areas were analyzed using a color imaging morphometry system (WinROOF, Mitani, Fukui, Japan). ${ }^{15}$

\section{In Vitro Blood Magnetic Resonance Imaging}

We investigated the contribution of blood contents or blood coagulation to DWI findings, using MRI in vitro. The Ethics Committee of University of Miyazaki and Koga General Hospital approved the protocol of the study in vitro (University of Miyazaki Study protocol 0-0665, Koga General Hospital Approval no. 19-23). We prepared platelet-rich plasma (PRP), whole blood (WB), and erythrocyte-rich blood (ERB; with plasma removed from centrifuged blood) from healthy 
volunteers, and assessed the blood samples by 1.5-T superconducting system using an eight-channel knee-phased array coil (Signa Excite HDxt; GE Medical Systems) under coagulated and noncoagulated conditions in vitro (-Supplementary Fig. S2, available in the online version). We calculated the SI ratio of the blood to that of the adjacent agarose gel phantom in DWI, T1WI, and T2WI, and ADC values in ADC maps ( $n=9$ each).

\section{Statistical Analysis}

All data are presented as medians and ranges or interquartile ranges or as means \pm standard deviation. Differences for individual groups were tested using Mann-Whitney $U$ tests or one-way analysis of variance with multiple comparison tests (GraphPad Prizm 5.01, GraphPad Software Inc., San Diego, California, United States). Relationships between parameters were analyzed using Spearman's rank correlation coefficients. Values with $p<0.05$ were regarded as statistically significant.

\section{Results}

Clinical and MRI Characteristics of Patients with DVT - Table 1 shows the clinical and MRI characteristics of the patients with DVT. Six (75\%) of them had lower extremity edema, swelling, redness, or stasis dermatitis. Contrast-enhanced chest CT showed that four (50\%) patients were compli-

Table 1 Baseline and MRI characteristics of patients with DVT

\begin{tabular}{|l|l|}
\hline & Total $(\boldsymbol{n}=\mathbf{8})$ \\
\hline Clinical characteristics & \\
\hline Median age (range; $y)$ & $62.5(24-88)$ \\
\hline Male, $n$ (\%) & $3(38)$ \\
\hline Symptomatic lower extremities, $n$ (\%) & $6(75)$ \\
\hline $\begin{array}{l}\text { Median elapsed time after } \\
\text { onset (range; } d)\end{array}$ & $12(0-60)$ \\
\hline Median D-dimer (range; $\mu \mathrm{g} / \mathrm{mL})$ & $7.8(1.1-172.5)$ \\
\hline Malignancy, $n$ (\%) & $2(25)$ \\
\hline Post-surgery, $n$ (\%) & $1(12.5)$ \\
\hline Chemotherapy, $n$ (\%) & $1(12.5)$ \\
\hline Previous DVT history & $2(25)$ \\
\hline Complications of PE & $4(50)$ \\
\hline MRI characteristics & \\
\hline Location of DVT & \\
\hline IVC and pelvic to femoral vein & $2(25)$ \\
\hline Pelvic to lower leg vein & $1(12.5)$ \\
\hline Pelvic vein & $1(12.5)$ \\
\hline Lower leg vein & $4(50)$ \\
\hline Both sides & $4(50)$ \\
\hline DVT volume & \\
\hline Total, median (range; mL) & $19.1(0.8-61.8)$ \\
\hline IVC and pelvis, median (range; mL) & $7.8(0-45.3)$ \\
\hline
\end{tabular}

Abbreviations: DVT, deep vein thrombosis; IVC, inferior vena cava; MRI, magnetic resonance imaging; $\mathrm{PE}$, pulmonary embolism. cated with small pulmonary embolism, without chest pain or respiratory symptoms. The D-dimer levels of all patients exceeded the cut-off value $(1.0 \mu \mathrm{g} / \mathrm{mL})$. The thrombi on T1WI occupied the IVC and the pelvic to the femoral vein $(n=2)$, the pelvic to the lower leg vein $(n=1)$, only the pelvic vein $(n=1)$, the lower leg vein $(n=4)$, both sides $(n=4)$, and the left $(n=3)$ and right $(n=1)$ sides. Two of the patients had previous DVT history. Two of the patients were incidentally detected DVT in investigation for other disease. The median DVT volume measured on T1WI was $19.1 \mathrm{~mL}$ (range, $0.8-61.8 \mathrm{~mL}$ ). The DVT volume correlated negatively with elapsed time after onset, $(r=-0.93, p=0.02, n=6)$. The DVT volume correlated positively with plasma D-dimer $(r=0.86, p=0.01, n=8)$. The detailed clinical characteristics of individual patients are shown in -Supplementary Table $\mathbf{S 2}$ (available in the online version). The extent of thrombi on MRI was concordant with that on ultrasound or CT in four cases. Two of three IVC thrombi were detected by only MRI. MRI detected five of six bilateral leg thrombi, while ultrasound and/or CT detected four of six thrombi.

- Fig. 1 A shows representative MR images of a patient with DVT. We analyzed CNR on DWI, T1WI, and T2WI, and the ADC of thrombi in axial images to determine whether MR findings of DVT differ among anatomical regions and between the proximal and distal portions of the DVT. - Fig. 1B shows the CNR or values of each MR sequence among the regions and between the DVT portions. The thrombus CNRs on DWI in IVC and pelvic region were significantly higher than those in femoral or lower leg regions. The CNRs were significantly higher in the proximal, than the distal portions of thrombi. The ADCs in IVC and pelvic region were significantly lower than those in femoral or lower legs. The ADCs were significantly lower in proximal, than the distal portions of the thrombi. The CNR on T1WI did not significantly differ among the anatomical regions and between the portions. The CNRs on T2WI were significantly higher in the IVC and pelvic region than in the femoral region. In contrast, the CNR on T2WI did not differ between the proximal and distal portions.

\section{Signal Changes in Venous Thrombus on DWI in Rabbit Model of DVT}

We assessed the rabbit model of DVT using MRI. We analyzed the CNR and ADC of jugular vein thrombi in axial images at 4 hours, and at 1, 2, and 3 weeks (-Fig. 2). The volumes of rabbit jugular vein thrombi measured using T1WI decreased over time (-Supplementary Fig. $\mathbf{5 3}$, available in the online version). The DWI CNRs of the thrombi at 4 hours were significantly higher than those at 1,2 , and 3 weeks, and the ADCs of the thrombi at 4 hours were significantly lower than those at 1, 2, and 3 weeks. The CNR of the thrombi on T1WI showed a peak SI at 1 week, and that on T2WI showed higher SI at 4 hours than at 3 weeks.

\section{Pathological Changes of Rabbit Jugular Vein Thrombus over the Time}

To determine relationships between DWI and thrombus organization, we pathologically and immunohistochemically assessed rabbit jugular thrombi corresponding to thrombotic 


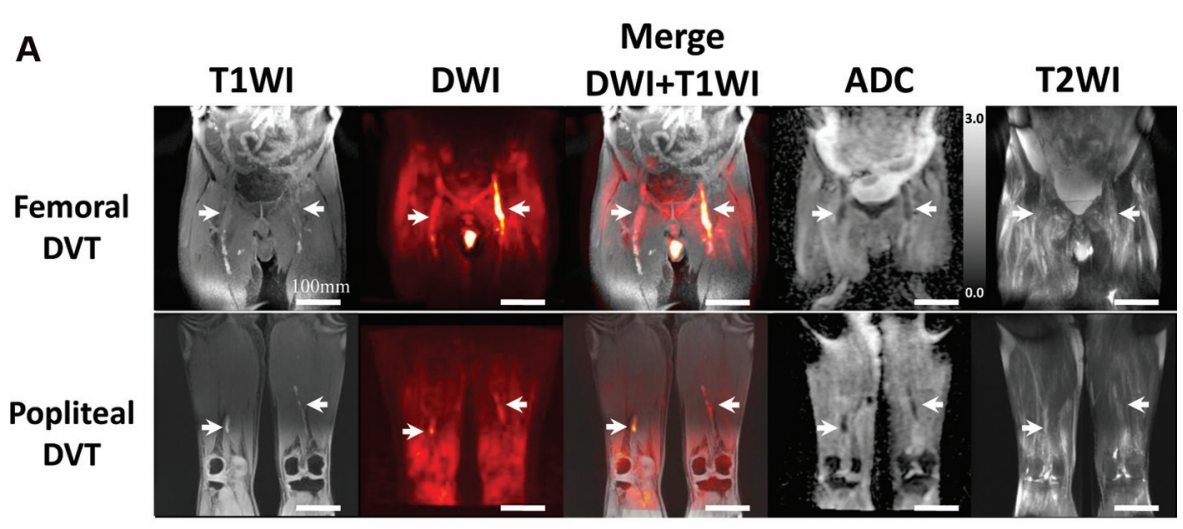

B
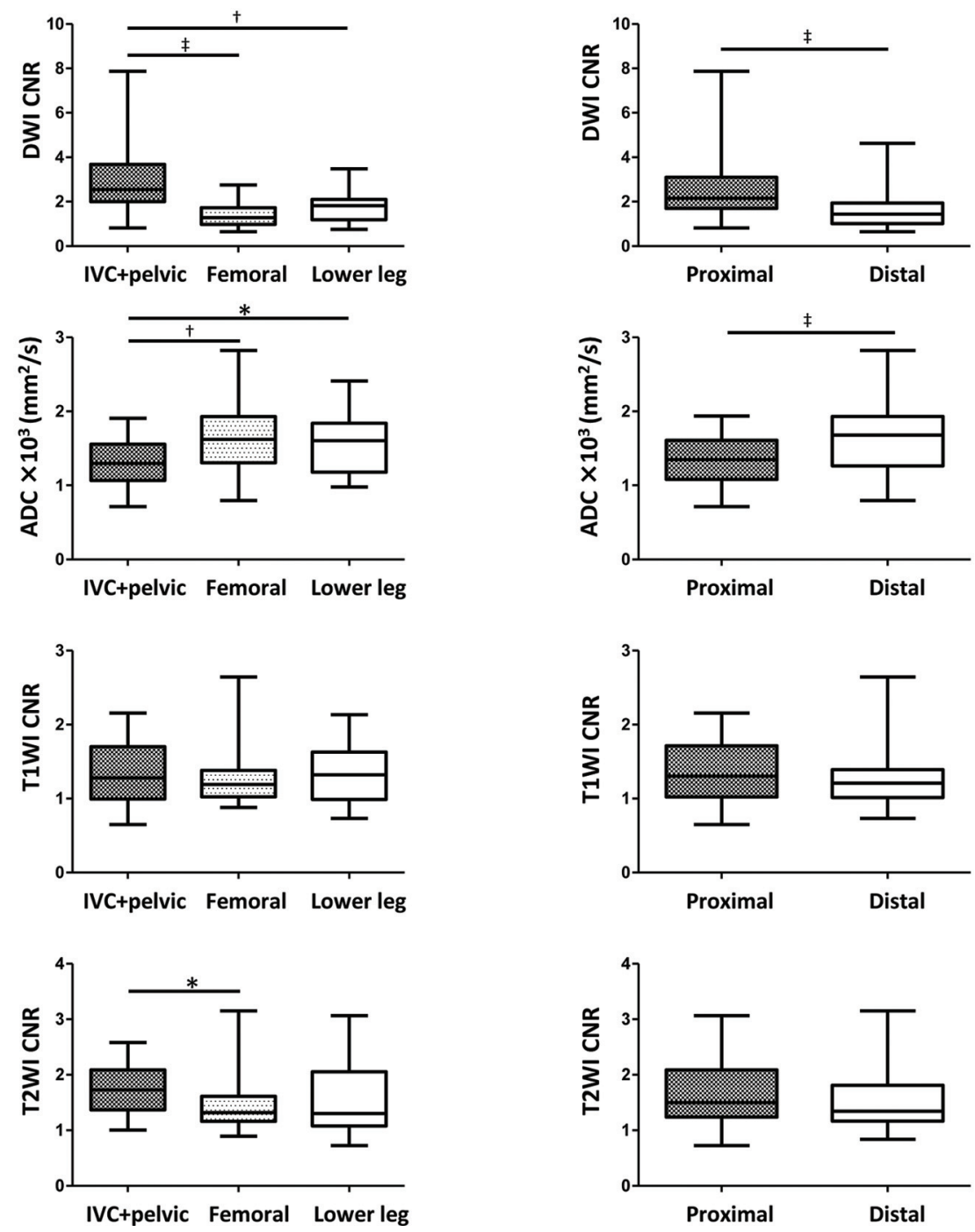

Fig. 1 MR imaging of human DVT. (A) Representative MR image of a patient with DVT. High SI or high-to-iso SI lesions on T1WI occupy bilateral femoral to popliteal veins (arrows), indicating DVT. Merged DWI and T1WI localize heterogeneous high-to-iso SI lesions in deep veins on DWI. Areas of DVT with high SI on DWI have low ADC on maps and high-to-iso SI on T2WI. Portions of DVT on DWI with high SI also have iso-to-high SI on T2WI. (B) CNR and ADC in IVC and pelvic $(n=35)$, femoral $(n=36)$, and lower leg $(n=32)$ portions of DVT, CNR, and ADC in proximal $(n=51)$ and distal $(n=52)$ portions of DVT. ${ }^{*} p<0.05,{ }^{\dagger} p<0.01,{ }^{\ddagger} p<0.001$ (Kruskal-Wallis tests with Dunn multiple comparison tests or Mann-Whitney $U$ tests). ADC, apparent diffusion coefficient; CNR, contrast noise ratio; DVT, deep vein thrombosis; DWI, diffusion-weighted image; IVC, inferior vena cava; MR, magnetic resonance; SI, signal intensity; T1WI, T1-weighted image; T2WI, T2-weighted image. 
lesions in axial MR images. Macroscopically, the thrombus was the largest at 4 hours, and became smaller over time. The thrombus was dark red at 4 hours and gradually changed to brown, then to yellow from the wall side (-Supplementary Fig. S4, available in the online version). Thrombi at 4 hours comprised erythrocytes, while platelets and fibrin were considered as fresh thrombus. Smooth muscle proliferation and macrophage infiltration, as well as iron and collagen deposition at the thrombus-venous wall interface were evident at 1 and 2 weeks, indicating organizing thrombus. Thrombi were replaced at 3 weeks by fibrous connective tissue, indicating organized thrombi (-Fig. 3A). - Fig. 3B shows areas of thrombus components and their change over time. Semiquantitative analysis of the images revealed timedependent decreases in areas of erythrocytes, and timedependent increases in areas with macrophages, collagen, and iron. Platelets and fibrin persisted for up to 2 weeks, and median platelet and fibrin contents peaked at 1 week. Smooth muscle cells (SMCs) also appeared at 1 week and the median numbers peaked 1 week later.

\section{Correlations between MR Sequences and Areas of Histopathological Thrombus}

We analyzed correlations between values on MR sequences and histopathologically confirmed areas of thrombus in the rabbit jugular vein to determine which factors affect DWI

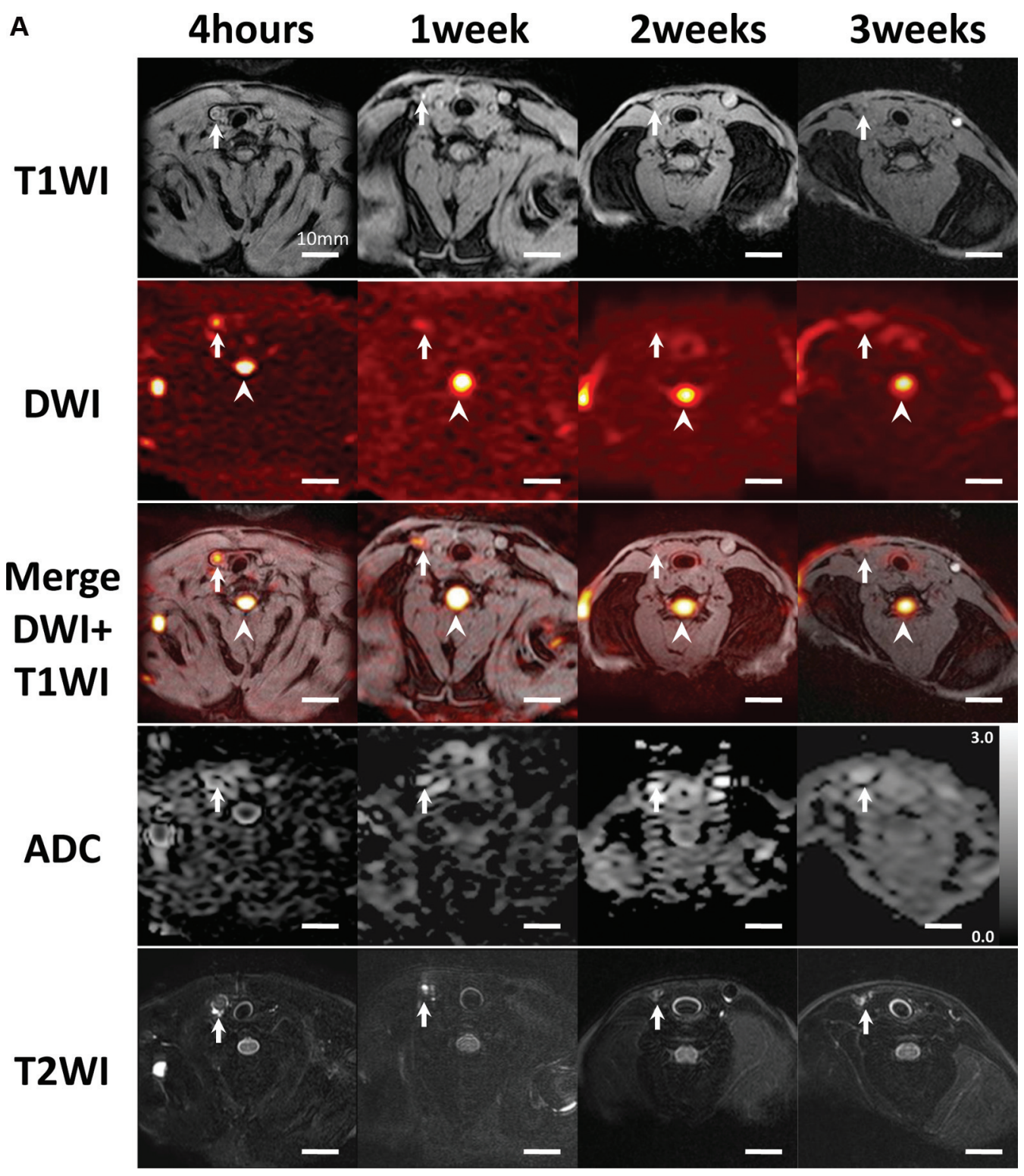

Fig. 2 Time-dependent changes of MR images of thrombi in rabbit jugular vein. (A) Lesions have high SI on DWI in right side of neck (arrows) at 4 hours and 1 week. Merged DWI and T1WI show lesions with high SI on DWI of right jugular veins. Thrombi appear on ADC maps as having relative iso values at 4 hours, and as higher values at 1, 2, and 3 weeks. Thrombi appear on T2WI, as slightly high SI at 4 hours, high SI at 1 week, and high-to-iso at 2 and 3 weeks. Spinal cord appears high SI on DWI and merged DWI and T1WI (arrowhead). (B) Changes in CNR of DWI, T1WI, and T2WI, and ADC in rabbit jugular vein thrombi over time, ${ }^{*} p<0.05,{ }^{\dagger} p<0.01,{ }^{\ddagger} p<0.001, n=28$ (4 hours), 20 (1 week), 15 ( 2 weeks), and 16 (3 weeks) (Kruskal-Wallis tests with Dunn multiple comparison tests). ADC, apparent diffusion coefficient; CNR, contrast noise ratio; DWI, diffusion-weighted image; MR, magnetic resonance; SI, signal intensity; T1WI, T1-weighted image; T2WI, T2-weighted image. 

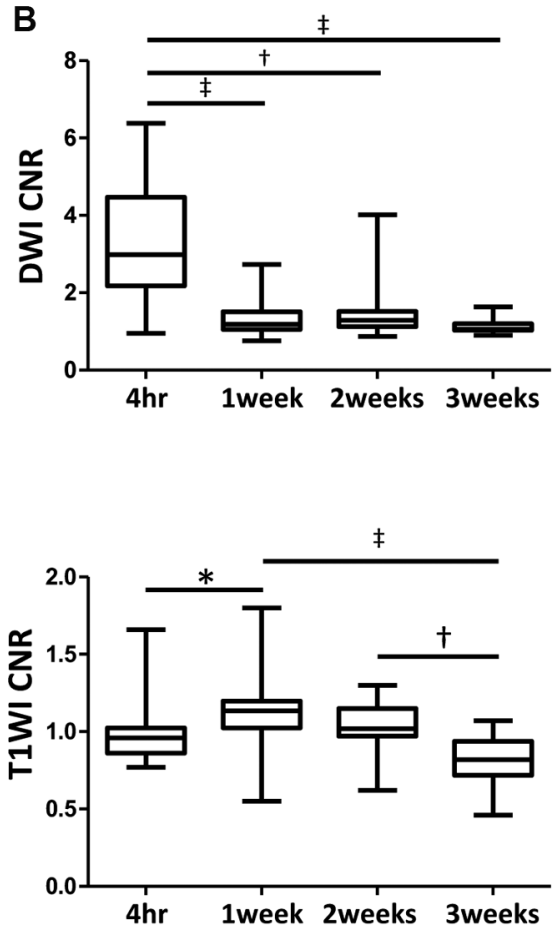

Fig. 2 (Continued)

image. - Supplementary Table $\mathbf{5 3}$ (available in the online version) shows relationships between the MR sequences and the histopathological parameters by the WinROOF color imaging morphometry system. The DWI CNR correlated positively with erythrocytes, weakly with platelets and fibrin, and negatively with SMC, macrophages, iron, and collagen content. Conversely, ADC correlated positively with SMC, macrophages, iron, and collagen content, and negatively with erythrocytes. The CNR on T1WI correlated weakly, but positively with fibrin and platelets, that on T2WI correlated weakly with erythrocytes.

\section{Human Blood Components with and without Coagulation Assessed In Vitro Using MRI}

The above correlations suggested that erythrocyte and/or platelet-fibrin contents affect DWI. We determined whether blood components and/or blood coagulation affect DWI and ADC using MRI in vitro of human WB, PRP, and ERB with or without coagulation. - Table 2 shows the cell counts of $\mathrm{WB}$, PRP, and ERB prepared by centrifugation. We assessed coagulated and noncoagulated blood and plasma on DWI, and the ADC of axial image (- Supplementary Fig. S5, available in the online version). - Fig. 4 shows the CNR and ADC of the coagulated blood. The blood SI (except for that of PRP) on DWI increased dependently on hematocrit, whereas the ADC of blood decreased dependently on hematocrit. The SI of PRP was the highest among the processed blood samples on T1WI and T2WI. The hematocrit did not affect SI of WB, ERB 1 (hematocrit, 58\%), and ERB 2 (hematocrit, 79\%) on T1WI and T2WI. The ADC did not differ between coagulated and noncoagulated blood (-Supplementary Fig. S6, available in the online version).
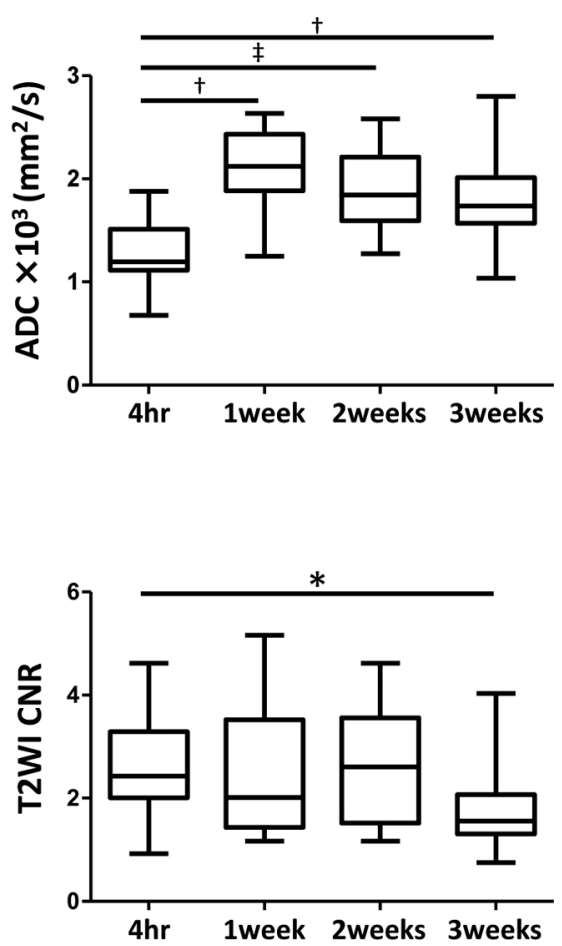

\section{Discussion}

We showed that MR-DWI can noninvasively detect human DVT and that the SI of DVT was higher in the proximal than the distal portion on DWI. The SI of thrombus in the rabbit jugular vein was high on DWI during the acute phase, and correlated positively with erythrocyte areas. In vitro blood MRI revealed a hematocrit-dependent increase in the SI on DWI and decrease in ADC.

The SI of human DVT was heterogeneously high to iso on DWI. Information about DWI findings of DVT is scant. Nakahashi et al assessed four patients with DVT and found high SI of venous thrombi on DWI, which disappeared after 1 year. ${ }^{16} \mathrm{Wu}$ et al reported that acute DVT ( $\leq 14$ days from clinical onset) appeared as hyperintensity lesion on DWI compared with nonacute DVT. ${ }^{17}$ We also reported that DWI detected DVT as a region of high SI in a symptomatic patient. ${ }^{18}$ The present findings are consistent with these previous reports. Favrole et al found high SI on DWI in $41 \%$ of patients with recent cerebral venous thrombosis and no high SI in any thrombi on follow-up MRI. ${ }^{19}$ In addition to high SI portions, DVT included iso SI on DWI. Fresh venous thrombus consists of erythrocytes, platelets, leukocytes, and fibrin that are gradually replaced by inflammatory cells, SMCs, and fibrous tissue. ${ }^{13,20}$ In fact, the organizing process of DVT in the lower extremities of humans goes through various phases, suggesting repeated thrombus formation. ${ }^{1,21}$ Our animal study revealed sequential change on DWI and histology of DVT. Therefore, the heterogeneity of DVT on DWI might be derived from admixture of fresh and organizing components, and the transition from high to iso SI on follow-up MRI might reflect organized thrombus. A follow-up imaging study of DVT by Lohr et al found that 53 (28\%) of 192 
patients had thrombus propagated by the initial DVT. ${ }^{22}$ The proximal high SI of human DVT on DWI suggested fresh components within the DVT. Although DWI can be affected by $\mathrm{T} 2 \mathrm{WI},{ }^{23}$ the DWI and T2WI findings of human DVT were not always similar. Anatomical differences in DVT were detectable in DWI and ADC, but not in T2WI.

The ADCs of rabbit jugular vein thrombi at 4 hours were significantly lower than those at 1,2, and 3 weeks. Only two studies have examined sequential changes in animal models of DVT on DWI. The ADCs were lower in erythrocyte-rich thrombus at day 1 and in collagen-rich organized thrombus at day 28 in a mouse IVC thrombi. ${ }^{24}$ The porcine IVC thrombus showed lower ADC in erythrocyte-rich thrombus with marginal neutrophils at day 1 , whereas the ADCs were higher in the erythrocyte-rich thrombus at 2 hours and organizing thrombus at day $14 .{ }^{25}$ Therefore, the sequential changes in the ADC of the DVT models are inconsistent. Except for the ADC at 2 hours in a previous study, ${ }^{25}$ the ADC of erythrocyte-rich thrombi at 4 hours and on day 1 was consistently low. In addition, we found a positive correlation between ADC and erythrocyte content. Therefore, the erythrocyte content in DVT might influence the ADC and could explain why the SI of DVT appeared high on DWI during the acute phase.

The results of the study in vitro support the notion that erythrocyte content affects the ADC and might restrict water diffusion. We presumed that fibrin gel formation in DVT affects water diffusion and its ADC. However, our data suggested that blood clotting and platelet aggregation during venous thrombus formation do not affect $A D C$. On the other hand, the high SI of PRP on DWI might have been due to a T2 shine-through effect rather than restricted water diffusion because the ADC of PRP is high. ${ }^{23}$ Considering all the above, a

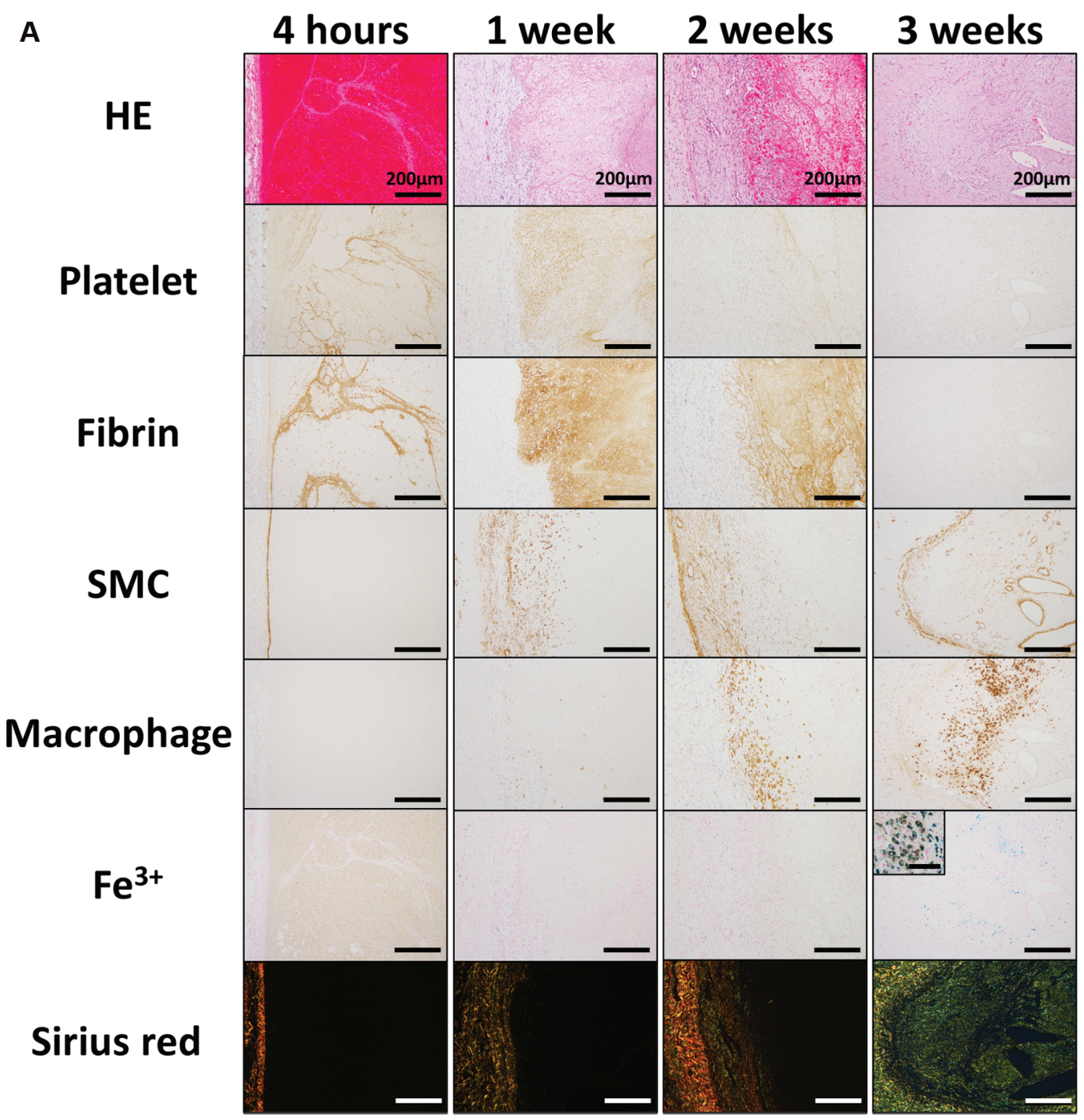

Fig. 3 Time-dependent histological changes in rabbit jugular venous thrombi. (A) Representative microphotographs of thrombi at 4 hours, and 1,2 , and 3 weeks. Thrombi at 4 hours are rich in erythrocytes, platelets, and fibrin without organizing process, and are considered as fresh. Proliferation of SMC, macrophage infiltration, and deposition of iron and collagen in thrombus and venous wall interface at 1 and 2 weeks, are considered as organizing thrombus. At 3 weeks, thrombus consists of fibrous connective tissue with SMC, macrophages, collagen, and recanalized capillaries. Iron is localized in macrophages, indicating hemosiderin deposition (inset bar in Fe ${ }^{3+}$ at 3 weeks indicates $50 \mu \mathrm{m}$ ). Furthermore, erythrocytes, platelets, and fibrin are scant, and thrombus is considered as organized. (B) Immunopositive, iron and collagen deposited areas of rabbit jugular vein thrombi at 4 hours, 1,2 , and 3 weeks. ${ }^{*} p<0.05,{ }^{\dagger} p<0.01,{ }^{\ddagger} p<0.001, n=28$ (4 hours), 20 (1 week), 15 ( 2 weeks), and 16 ( 3 weeks) (Kruskal-Wallis tests with Dunn multiple comparison tests). HE, hematoxylin and eosin; SMC, smooth muscle cells. 

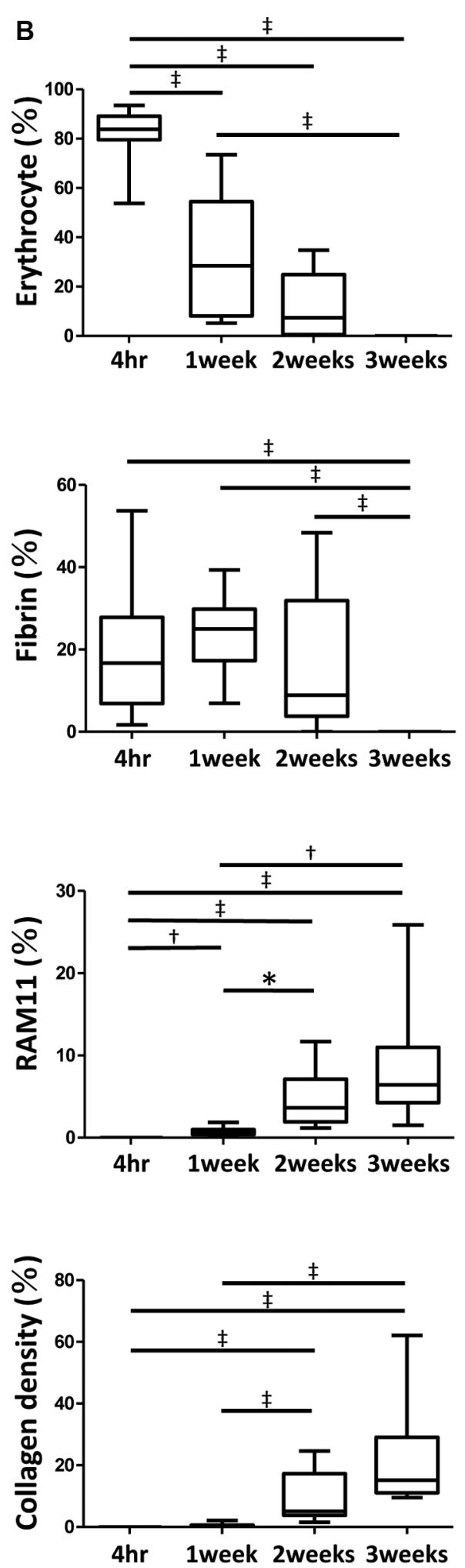

Fig. 3 (Continued)

proximal high SI of human DVT accompanied by a low ADC might reflect an erythrocyte-rich, fresh component within a deep vein thrombus.

DWI relies on detecting the random motion of free water molecules. Although our results suggest that erythrocyte content restricts water diffusion during the acute phase of
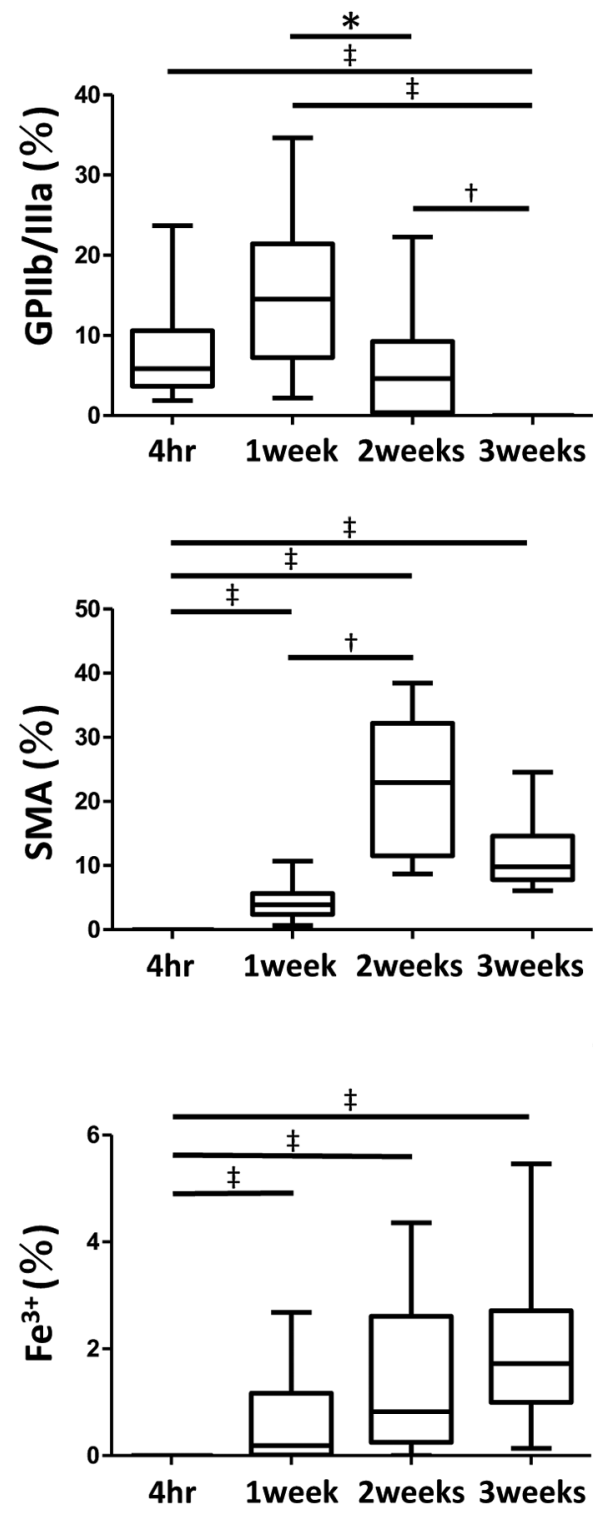

DVT, the mechanisms remain unknown. Methemoglobin and a hypercellular environment could restrict water diffusion in DVT. Water molecules can interact with the ferric center in methemoglobin, ${ }^{26}$ a hemoglobin derivative, and the interaction might restrict the diffusion of water molecules. The motion of water molecules could be impeded by interactions 
Table 2 Differential counts in human venous blood after centrifugation

\begin{tabular}{|l|l|l|l|l|}
\hline Counts $(\mathrm{means} \pm \mathrm{SD})$ & PRP & Whole blood & ERB 1 & ERB 2 \\
\hline RBC $\left(10^{6} / \mu \mathrm{L}\right)$ & $0.01 \pm 0.003$ & $4.20 \pm 0.20$ & $6.7 \pm 0.1$ & $8.9 \pm 0.1$ \\
\hline $\mathrm{Ht}(\%)$ & $0.1 \pm 0$ & $37 \pm 2$ & $58 \pm 0.3$ & $79 \pm 0.1$ \\
\hline Plt $\left(10^{3} / \mu \mathrm{L}\right)$ & $447 \pm 20$ & $225 \pm 43$ & $80 \pm 6$ & $73 \pm 5$ \\
\hline WBC $(/ \mu \mathrm{L})$ & $40 \pm 40$ & $5,500 \pm 600$ & $8,500 \pm 500$ & $7,300 \pm 700$ \\
\hline
\end{tabular}

Abbreviations: ERB, erythrocyte-rich blood; Ht, hematocrit; Plt, platelet; PRP, platelet-rich plasma; RBC, red blood cells; SD, standard deviation; WBC, white blood cells.
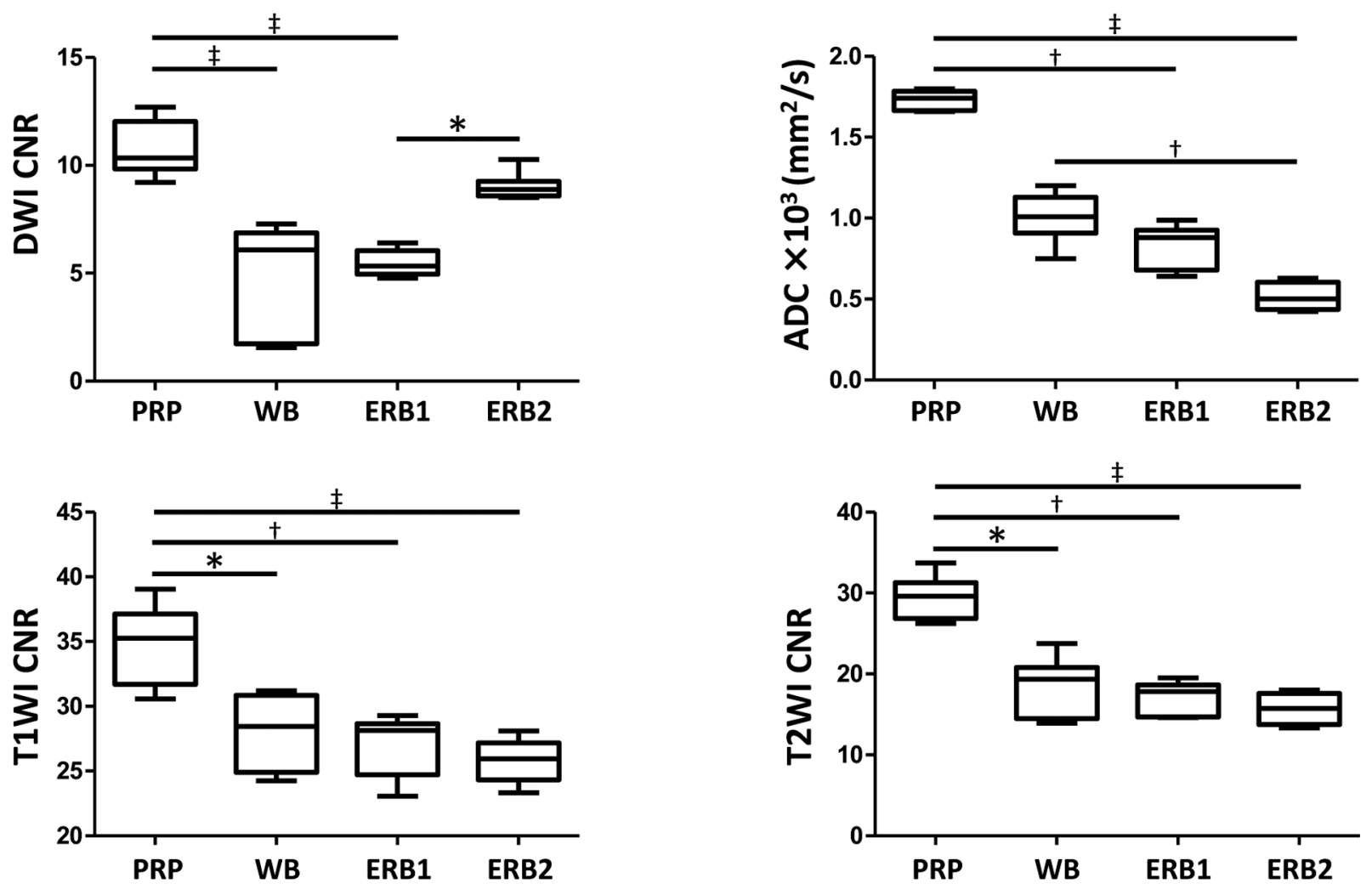

Fig. 4 MR imaging of coagulated blood in vitro. Human venous blood containing sodium citrate was separated into WB (Ht $37 \%$ ), PRP (Ht $0.1 \%)$, and ERB 1 ( $\mathrm{Ht} 58 \%$ ) and 2 ( $\mathrm{Ht} 79 \%$ ). Blood was coagulated using human placental tissue factor and $\mathrm{CaCl}_{2}$ solution. CNR of DWI, T1WI, and T2WI, and ADC of coagulated blood in vitro. ${ }^{*} p<0.05,{ }^{\dagger} p<0.01,{ }^{\ddagger} p<0.001, n=9$ per processed blood sample (Kruskal-Wallis tests with Dunn multiple comparison tests). ADC, apparent diffusion coefficient; CNR, contrast-to-noise ratios; DWI, diffusion-weighted imaging; ERB, erythrocyte-rich blood; $\mathrm{Ht}$, hematocrit; MR, magnetic resonance; PRP, platelet-rich plasma; T1WI, T1-weighted image; T2WI, T2-weighted image; WB, whole blood.

with cell membranes and/or extracellular matrix, and reduced extracellular space in the hypercellular environment of malignant tumor. ${ }^{27}$ Venous thrombus during the acute phase consists mostly of erythrocytes, which implies a hypercellular environment.

High accuracy of ultrasound and CT for diagnosis of DVT in lower extremities has been reported. ${ }^{5}$ Additionally, previous studies showed that MR direct thrombus imaging, based on T1WI, can detect recurrent $\mathrm{DVT}^{8-10}$ and accurately detect pelvic vein thrombosis in pregnancy. ${ }^{28}$ In our study, IVC/pelvic thrombi, incidental, and recurrent DVT were detected by MRI. In addition, a clinical and a basic imaging study tried to assess thrombus age. Multiparametric MRI (combined analysis using T2W1 and ADC) was an efficient method for discriminating among venous thrombi, acute, and chronic pulmonary thromboemoboli with autopsy samples. ${ }^{29}$ ${ }^{18} \mathrm{~F}$-fluorodeoxyglucose positron emission tomography/CT identified jugular vein venous thrombus and a time-depending signal change in mice. ${ }^{30}$ The present study showed MRDWI is a possible tool of determination for venous thrombus age based on erythrocyte content. Therefore, MRI may have an important role as a complementary imaging tool, especially in recurrent, incidental, IVC/pelvic DVT, during pregnancy, and thrombus age assessment.

This study has several limitations. The study cohort comprised only eight patients; thus, a larger clinical study is needed to assess the ability of DWI to diagnose DVT. Besides, five of these patients were treated with anticoagulant administration before MRI. Anticoagulant therapy might affect thrombus components, especially in fibrin contents, because fibrin content was correlated with DWI and T1WI CNRs in this study. We were unable to evaluate the histopathological findings of human DVT and thus extrapolated clinical findings from experimental animals and studies in vitro. 


\section{Conclusion}

DWI can detect venous thrombus, and high SI on DWI accompanied by a low ADC might reflect erythrocyte-rich, acute-phase thrombi.

\section{What is known about this topic?}

- The effects of antithrombotic therapy on deep vein thrombosis can be affected by thrombus age.

- Reliable noninvasive imaging to evaluate thrombus age have not been established.

\section{What does this paper add?}

- Acute-phase venous thrombi were depicted as high signal intensity on magnetic resonance diffusionweighted image in human and rabbit venous thrombus.

- The signal intensity of diffusion-weighted image may reflect erythrocyte content in rabbit venous thrombus and in vitro blood.

\section{Funding}

This study was partly supported by Grants-in-Aid for Scientific Research (Nos. JP15K09963, JP16K09019, JP18K15083, JP19K07437, JP19H03445, JP20K08085) from Japan Society for the Promotion of Science, the President's Strategic Priority Budget from Miyazaki University, Clinical Research from Miyazaki University Hospital, and a Research Promotion Grant from The Japanese Society on Thrombosis and Hemostasis.

\section{Conflict of Interest}

None declared.

\section{Acknowledgments}

The authors are grateful to Ritsuko Sotomura, Kyoko Ohashi, Nahoko Udatsu, Masahiro Enzaki, Chihiro Iida, Yugo Kashiwagi, and Sora Hanzawa for excellent technical assistance. We are also grateful to Dr. Kazuo Umemura and Dr. Nobuo Nagai for valuable discussions.

\section{References}

1 Furukoji E, Gi T, Yamashita A, et al. CD163 macrophage and erythrocyte contents in aspirated deep vein thrombus are associated with the time after onset: a pilot study. Thromb J 2016;14:46

2 Marder VJ, Sherry S. Thrombolytic therapy: current status (2). N Engl J Med 1988;318(24):1585-1595

3 Francis CW, Marder VJ. Fibrinolytic therapy for venous thrombosis. Prog Cardiovasc Dis 1991;34(03):193-204

4 Gersh KC, Nagaswami C, Weisel JW. Fibrin network structure and clot mechanical properties are altered by incorporation of erythrocytes. Thromb Haemost 2009;102(06):1169-1175

5 Karande GY, Hedgire SS, Sanchez Y, et al. Advanced imaging in acute and chronic deep vein thrombosis. Cardiovasc Diagn Ther 2016;6(06):493-507
6 Fraser DG, Moody AR, Morgan PS, Martel AL, Davidson I. Diagnosis of lower-limb deep venous thrombosis: a prospective blinded study of magnetic resonance direct thrombus imaging. Ann Intern Med 2002;136(02):89-98

7 Kelly J, Hunt BJ, Moody A. Magnetic resonance direct thrombus imaging: a novel technique for imaging venous thromboemboli. Thromb Haemost 2003;89(05):773-782

8 Tan M, Mol GC, van Rooden CJ, et al. Magnetic resonance direct thrombus imaging differentiates acute recurrent ipsilateral deep vein thrombosis from residual thrombosis. Blood 2014;124(04):623-627

9 Dronkers CEA, Klok FA, van Langevelde K, et al. Diagnosing recurrent DVT of the leg by two different non-contrast-enhanced magnetic resonance direct thrombus imaging techniques: a pilot study. TH Open 2019;3(01):e37-e44

10 van Dam LF, Dronkers CEA, Gautam G, et al. Magnetic resonance imaging for diagnosis of recurrent ipsilateral deep vein thrombosis. Blood 2020;135(16):1377-1385

11 Yamaki T, Nozaki M, Sakurai H, et al. Combined use of pretest clinical probability score and latex agglutination D-dimer testing for excluding acute deep vein thrombosis. J Vasc Surg 2009;50 (05):1099-1105

12 Rosset A, Spadola L, Ratib O. OsiriX: an open-source software for navigating in multidimensional DICOM images. J Digit Imaging 2004;17(03):205-216

13 Kuroiwa Y, Yamashita A, Miyati T, et al. MR signal change in venous thrombus relates organizing process and thrombolytic response in rabbit. Magn Reson Imaging 2011;29(07):975-984

14 Yamashita A, Matsuda S, MatsumotoT, et al. Thrombin generation by intimal tissue factor contributes to thrombus formation on macrophage-rich neointima but not normal intima of hyperlipidemic rabbits. Atherosclerosis 2009;206(02):418-426

15 Yamashita A, Shoji K, Tsuruda T, et al. Medial and adventitial macrophages are associated with expansive atherosclerotic remodeling in rabbit femoral artery. Histol Histopathol 2008;23 (02):127-136

16 Nakahashi M, Sato N, Tsushima Y, Amanuma M, Endo K. Diffusionweighted magnetic resonance imaging of the body in venous thrombosis: a report of four cases. Abdom Imaging 2008;33(03): 353-356

17 Wu G, Morelli J, Xiong Y, Liu X, Li X. Diffusion weighted cardiovascular magnetic resonance imaging for discriminating acute from non-acute deep venous thrombus. J Cardiovasc Magn Reson 2019;21(01):37

18 Kuroiwa Y, Aburaya M, Yamashita A, et al. Diffusion-weighted MR imaging of deep vein thrombosis. Magn Reson Med Sci 2016;15 (01):144-145

19 Favrole P, Guichard JP, Crassard I, Bousser MG, Chabriat H. Diffusion-weighted imaging of intravascular clots in cerebral venous thrombosis. Stroke 2004;35(01):99-103

20 Fineschi V, Turillazzi E, Neri M, Pomara C, Riezzo I. Histological age determination of venous thrombosis: a neglected forensic task in fatal pulmonary thrombo-embolism. Forensic Sci Int 2009;186(13):22-28

21 Ro A, Kageyama N, Tanifuji T, Fukunaga T. Pulmonary thromboembolism: overview and update from medicolegal aspects. Leg Med (Tokyo) 2008;10(02):57-71

22 Lohr JM, James KV, Deshmukh RM, Hasselfeld KA. Allastair B. Karmody Award. Calf vein thrombi are not a benign finding. Am J Surg 1995;170(02):86-90

23 Provenzale JM, Engelter ST, Petrella JR, Smith JS, MacFall JR. Use of MR exponential diffusion-weighted images to eradicate T2 "shine-through" effect. AJR Am J Roentgenol 1999;172(02): 537-539

24 Phinikaridou A, Andia ME, Saha P, Modarai B, Smith A, Botnar RM. In vivo magnetization transfer and diffusion-weighted magnetic resonance imaging detects thrombus composition in a mouse model of deep vein thrombosis. Circ Cardiovasc Imaging 2013;6 (03):433-440 
25 Ichiki M, Sakai Y, Nango M, et al. Experimental venous thrombi: MRI characteristics with histopathological correlation. Br J Radiol 2012;85(1012):331-338

26 Yi J, Thomas LM, Richter-Addo GB. Structure of human R-state aquomethemoglobin at $2.0 \AA$ resolution. Acta Crystallogr Sect $\mathrm{F}$ Struct Biol Cryst Commun 2011;67(Pt 6):647-651

27 Bonekamp S, Corona-Villalobos CP, Kamel IR. Oncologic applications of diffusion-weighted MRI in the body. J Magn Reson Imaging 2012;35(02):257-279

28 Torkzad MR, Bremme K, Hellgren M, et al. Magnetic resonance imaging and ultrasonography in diagnosis of pelvic vein thrombosis during pregnancy. Thromb Res 2010;126(02):107-112
29 Vidmar J, Kralj E, Bajd F, Serša I. Multiparametric MRI in characterizing venous thrombi and pulmonary thromboemboli acquired from patients with pulmonary embolism. J Magn Reson Imaging 2015;42(02):354-361

30 Hara T, Truelove J, Tawakol A, et al. 18F-fluorodeoxyglucose positron emission tomography/computed tomography enables the detection of recurrent same-site deep vein thrombosis by illuminating recently formed, neutrophil-rich thrombus. Circulation 2014;130(13):1044-1052 